\title{
High-Concentration Liquid Prednisolone Formula: Filling a Therapeutic Niche in Severe Acute Attacks of Urticaria and Angioedema
}

\author{
Adriane Peveling-Oberhag ${ }^{a}$ Holger Reimann $^{c}$ Veronika Weyer ${ }^{b}$ \\ Evgeny Goloborodko ${ }^{a}$ Petra Staubach ${ }^{a}$ \\ ${ }^{a}$ Department of Dermatology and ${ }^{b}$ Institute of Medical Biostatistics, Epidemiology and Informatics, University \\ Medical Center, Johannes Gutenberg University Mainz, Mainz, and 'Pharmaceutical Laboratory of the NRF, \\ Eschborn, Germany
}

\section{Key Words}

Corticosteroids · Urticaria · Angioedema

\begin{abstract}
Background/Aims: According to current guidelines, the emergency kit for patients with severe urticaria and/or angioedema should include a corticosteroid with a prednisoloneequivalent of 50-100 mg. Since severe dysphagia may occur in anaphylaxis, liquid corticosteroids are advantageous. Presently, only liquid preparations with less than 100 mg prednisolone equivalent are available worldwide. Methods: We prepared a highly concentrated liquid prednisolone formula for oral administration ( 1 or $5 \mathrm{mg}$ prednisolone per $\mathrm{ml}$ ). We observed efficacy and safety of $100 \mathrm{mg}$ or $>250 \mathrm{mg}$ liquid oral prednisolone in comparison to intravenous administration (250 mg prednisolone) in 53 patients with urticaria and/or angioedema. Results: The symptom control achieved with oral administration was comparable to that obtained with intravenous therapy, with remission of at least $50 \%$ of the symptoms in less than $30 \mathrm{~min}$. No side effects occurred during the treatment period. Conclusion: The liquid prednisolone formula is an additional therapeutic rescue medication in dermatological emergency medicine, filling a therapeutic niche for patients who need high-concentration liquid prednisolone. It is suitable for self-administration emergency kits in children and adults, in accordance with current guidelines.
\end{abstract}

(c) 2015 S. Karger AG, Basel

\section{KARGER}

E-Mail karger@karger.com

www.karger.com/spp

\section{Introduction}

Systemic corticosteroids are drugs with frequent application in emergency medicine. Interestingly, the skin contains its own glucosteroidogenic system $[1,2]$. According to anaphylaxis and urticaria guidelines [3-6], emergency kits for urticaria and/or angioedema patients have to contain, beside antihistamines, adrenaline, and $\beta$-receptor agonists, a self-administrable corticosteroid with a prednisolone equivalent of 50-100 $\mathrm{mg}$. In some patients with severe anaphylactic reactions, there is a need for high-concentration liquid prednisolone with more than $100 \mathrm{mg}$ prednisolone equivalent per dose. Since the drug should act fast and be easy to swallow, a liquid corticosteroid is favorable. Presently, only liquid preparations with less than $100 \mathrm{mg}$ prednisolone equivalent are available worldwide. Hence, the Department of Extemporaneous Preparations of the Society for Dermopharmacy in Germany [7] recommended establishing the clinical use of a new formula containing prednisolone up to $500 \mathrm{mg}$ per $100 \mathrm{ml}$ liquid agent. This expert panel gives recommendations for the use of extemporaneous preparations and is working closely with the Pharmaceutical Laboratory of the NRF, the national pharmacist compounding formulary in Germany. Their unlicensed pharmaceutical preparations for the specific needs of patients are habitually standardized for pharmacy prepara-

Assoc. Prof. Petra Staubach, MD

Department of Dermatology, University Medical Center Johannes Gutenberg University Mainz

Langenbeckstrasse 1, DE-55131 Mainz (Germany)

E-Mail petra.staubach@ unimedizin-mainz.de 
tion according to legislation. The new prednisolone formula was originally mentioned in The Netherlands in 1997 by the FNA, the Dutch pharmacist compounding formulary [8]. It contains prednisolone sodium phosphate with a prednisolone equivalent of up to $500 \mathrm{mg}$ per $100 \mathrm{ml}$ [9]. Prednisolone sodium phosphate is a watersoluble prodrug, but in vivo prednisolone is readily liberated by phosphases. Prednisolone is rapidly and well absorbed in the gastrointestinal tract following oral administration [10-15]. Its kinetics are nonlinear due to its nonlinear $70-90 \%$ plasma protein binding. Mean peak plasma concentrations occur between 40 and $55 \mathrm{~min}$, with a mean plasma half-life of about $2.9 \mathrm{~h}$ [10]. The peak plasma level is higher and occurs faster than with tablets [13-15]. Prednisolone is metabolized mainly in the liver and is excreted by the kidneys [11-15]. In bioavailability studies, pharmacokinetic parameters have indicated that the preparation is bioequivalent to tablets when corrected for dose $[10,11]$.

In the past, no controlled or noninterventional studies have been performed observing the effectiveness of different concentrations and therapeutic options of liquid corticosteroids in emergency situations. The aim of this prospective, noninterventional observational study was to prove the efficacy and safety of this high-concentration liquid prednisolone in emergency situations, e.g. urticaria with angioedema, in comparison to standard treatment with intravenous (i.v.) corticosteroids.

\section{Methods}

A total of 53 patients presenting as emergencies to our university hospital with anaphylaxis grades I and II were included. Symptoms comprised severe acute or exacerbated chronic spontaneous urticaria and/or angioedema combined with pruritus, dysphagia, and/or dyspnea. All patients were treated according to current guidelines [3-6]. After a physical examination and informed consent, all symptoms were recorded and judged separately, especially documenting the following: hives, angioedema, pruritus, dyspnea, and dysphagia. The location, size, number, severity, and duration of symptoms were documented on a standardized worksheet. The observed symptoms were categorized by the admitting physician as mild, moderate, or severe according to Common Terminology Criteria for Adverse Events (CTCAE) [16]. The examinations were approved by the local ethics committee.

We observed the efficacy of the liquid prednisolone formula at two different concentrations: a low dose (LD; $100 \mathrm{mg}$ prednisolone equivalent) and a high dose (HD; >250 mg), in comparison to i.v. application (250 mg) [8]. The LD consists of $100 \mathrm{ml}$ prednisolone oral solution, at a concentration of $1.34 \mathrm{mg}$ prednisolone sodium phosphate per milliliter, equivalent to $1 \mathrm{mg}$ prednisolone per milliliter. The HD is $100 \mathrm{ml}$ prednisolone oral solution, at a concen- tration of $6.72 \mathrm{mg}$ prednisolone sodium phosphate per milliliter, equivalent to $5 \mathrm{mg}$ prednisolone per milliliter.

The treatment mode was individually chosen by the physician and in case of i.v. administration it was also performed by the physician. Patients receiving the oral medication were instructed to drink either the whole bottle of $0.1 \%$ liquid prednisolone (LD) or half of the bottle of $0.5 \%$ liquid prednisolone (HD). The dose and mode of application, as well as all adverse events during treatment, were documented. Vital signs and skin status were monitored every $30 \mathrm{~min}$. In case of persisting symptoms, the dose was raised in both application forms. Every $30 \mathrm{~min}$ for up to $2 \mathrm{~h}$, the physician and patients were asked to make a symptom assessment. All patients were placed under surveillance for at least $2 \mathrm{~h}$. At discharge, the patients were asked to report recurring symptoms within the following days.

For statistical analysis, we performed a 2-tailed Mann-Whitney $\mathrm{U}$ test for comparison of LD and HD levels and used a repeatedmeasures ANOVA to detect differences between dose levels.

\section{Results}

We observed 53 patients ( 36 women and 17 men) aged 17-75 years (mean $47 \pm 15.6$ ) presenting as emergencies with anaphylactic symptoms. Overall, we recorded 180 symptoms including urticaria, pruritus, angioedema, dyspnea, dysphagia, and others. The primary presenting symptoms were urticaria and angioedema, and pruritus was the most common secondary symptom (fig. 1). Patients presented with 3.8 symptoms on average.

Ten patients received $250 \mathrm{mg}$ prednisolone i.v. and 43 patients received oral medication, i.e. HD liquid prednisolone (16 patients) or LD liquid prednisolone (27 patients). All patients, except one, showed either urticaria or angioedema at presentation. Regardless of the application mode (oral or i.v.), at least $50 \%$ of all symptoms disappeared within $30 \mathrm{~min}$ after treatment. HD of oral prednisolone were the most effective, with $70.4 \%$ of symptoms resolving within $30 \mathrm{~min}$ and only $22.2 \%$ persisting longer than $1 \mathrm{~h}$ (fig. 2a). Overall, treatment modes were comparable, with no significant differences in time to symptom control between the oral and i.v. groups $(p=0.0989)$. No relapse of symptoms was reported.

Subgroup analyses of the severity grades of symptoms indicated that severe symptoms needed higher doses of prednisolone liquid (fig. 2b). Especially in severe forms of urticaria and angioedema, the two most common presenting symptoms in dermatological emergencies, HD ( $\geq 250 \mathrm{mg}$ prednisolone liquid) showed superior symptom control (remission of $>70 \%$ in the first $30 \mathrm{~min}$ ) compared to $\mathrm{LD}(100 \mathrm{mg}, 50 \%$ remission, $<30 \mathrm{~min} ; \mathrm{p}=$ 0.008 ).
Peveling-Oberhag et al. 
Fig. 1. Before treatment, a total of 180 symptoms were presented by 53 patients receiving 3 different corticosteroid treatments: HD oral, LD oral, and i.v.

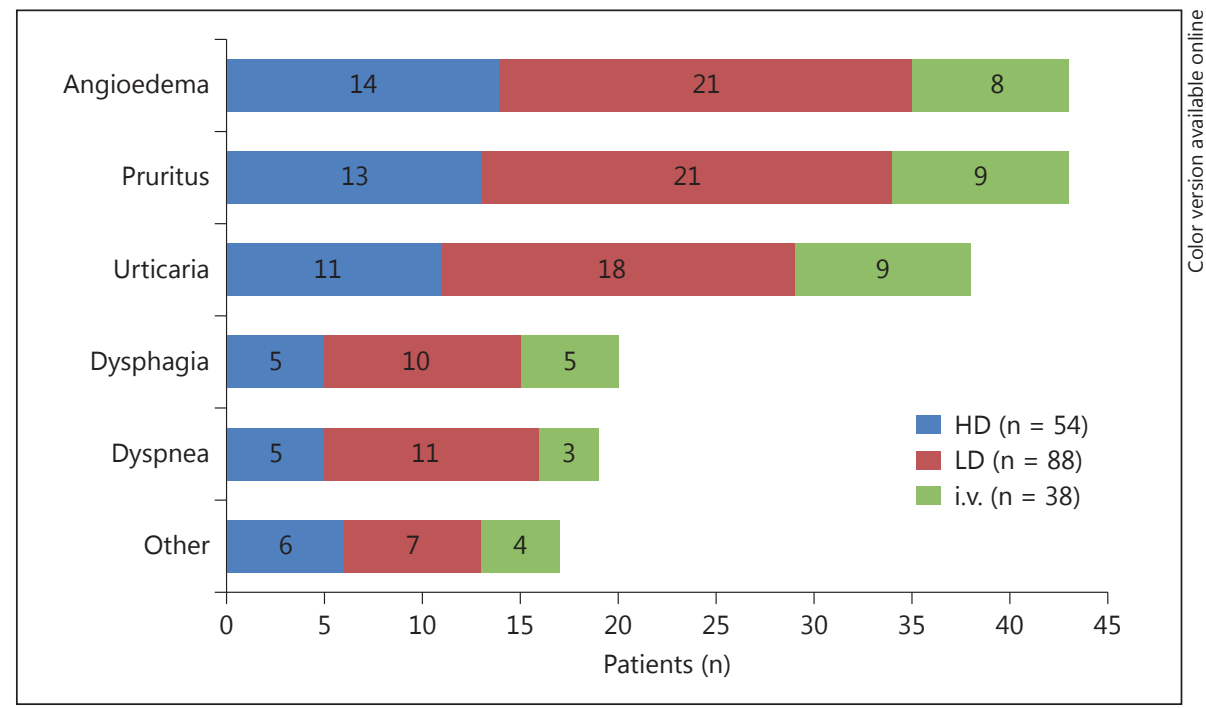

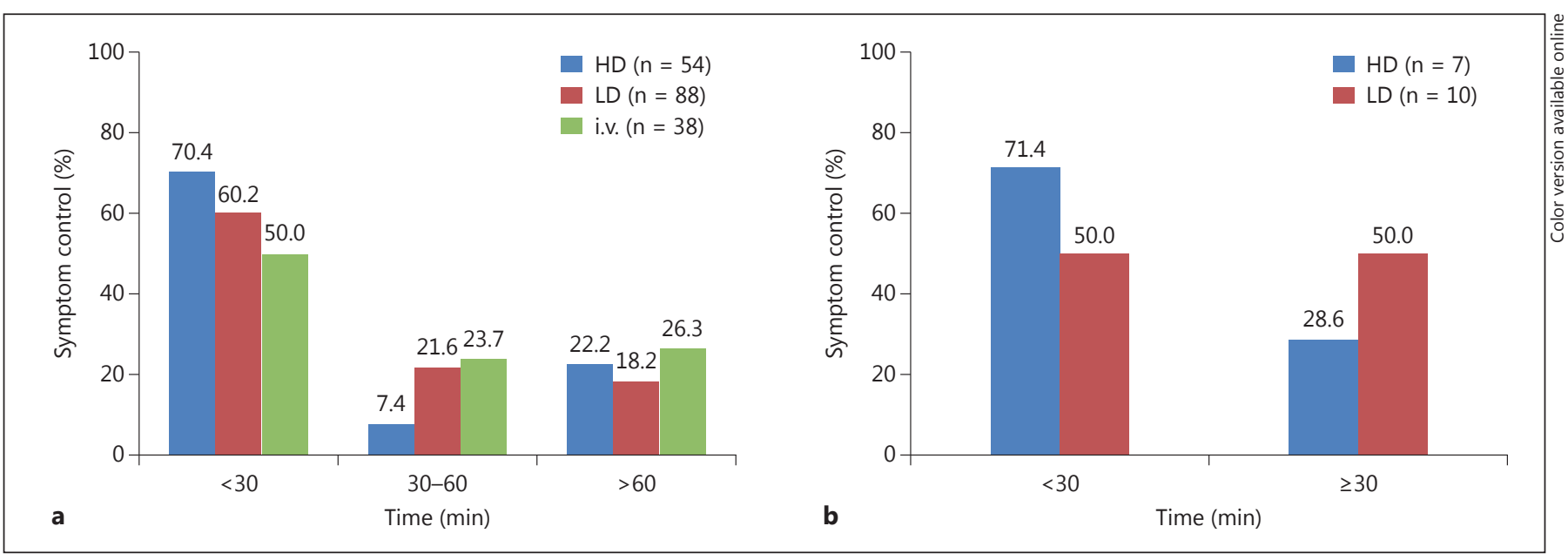

Fig. 2. a Overall symptom control. In all treatment groups (HD oral, LD oral, and i.v.) the majority of symptoms resolved within 30 min after treatment. b Severe-symptom control. Patients with severe forms of urticaria and angioedema benefited from HD of liquid prednisolone.

\section{Discussion}

According to urticaria guidelines, patients with severe urticaria attacks and/or angioedema combined with pruritus, dysphagia, and/or dyspnea must be treated with highly concentrated corticosteroids with a single dose or for up to several days as short-term therapy. All emergency kits should contain, besides other medications, a remedy with a prednisolone equivalent of $50-100 \mathrm{mg}$. The idea of using liquid corticosteroids for the treatment of urticaria and/or angioedema is not entirely new. How- ever, presently no such liquid formula is being marketed and studies reporting the efficacy of orally taken liquid corticosteroids are scarce. Lucas-Bouwman et al. [17] evaluated compliance and the effectiveness of prednisolone in asthmatic children, with the medication administered either as crushed tablets or as an oral solution. They found both drug formulas to be equally effective, but the liquid prednisolone was significantly better tolerated than the tablets. In the current study, we show the efficacy of a new treatment option for urticaria and/or angioedema combined with pruritus, dysphagia, and/or dys- 
pnea. This liquid prednisolone leads to rapid remission of symptoms with LD as well as with HD. Treatment of patients with the liquid prednisolone formula turned out to be as effective as standard emergency treatment, i.e. i.v. prednisolone $(250 \mathrm{mg})$. Patients with severe urticaria and/or angioedema (according to CTCAE criteria) may benefit from a higher concentration of liquid prednisolone ( $>250 \mathrm{mg}$ ). As patients were not randomized to either treatment group, there might be a possible selection bias limiting the interpretation of our data.

The general safety profile of liquid prednisolone is comparable to that of the tablet formula [13-15], and in the current study no adverse events occurred during administration of the drug.

Also the inclusion of oral prednisolone in self-administration emergency kits is conceivable, but to date there is little experience with such emergency medications outside hospital emergency departments.

We conclude that the new oral liquid prednisolone formula is a therapeutic option for treating anaphylactic symptoms in emergency situations, with the potential to substitute i.v. injection in selected cases. Updosing seems to be necessary in patients with acute severe urticaria and angioedema attacks with or without dyspnea/dysphagia. Larger, multicenter, comparative effectiveness trials are needed to determine the best dose of this new liquid prednisolone, including home treatment. Further therapeutic possibilities are treatment strategies especially in children, where tablets and the daily use of a suppository are best avoided.

\section{Disclosure Statement}

A. Peveling-Oberhag is or recently was a speaker for Roche and Novartis. H. Reimann, V. Weyer, and E. Goloborodko report no conflict of interests. P. Staubach is or recently was a speaker and/ or scientific advisor for Abbvie, Braun, Essex Pharma, MSD, Novartis, Pohl-Boskamp, Schering-Plough, Shire, and Viropharma.

\section{References}

1 Slominski AT, Manna PR, Tuckey RC: Cutaneous glucocorticosteroidogenesis: securing local homeostasis and the skin integrity. Exp Dermatol 2014;23:369-374.

2 Slominski A, Zbytek B, Nikolakis G, et al: Steroidogenesis in the skin: implications for local immune functions. J Steroid Biochem Mol Biol 2013;137:107-123.

3 Werner-Busse A, Zuberbier T, Worm M: The allergic emergency - management of severe allergic reactions. J Dtsch Dermatol Ges 2014; 12:379-387.

4 Zuberbier T, Aberer W, Asero R, et al: Methods report on the development of the 2013 revision and update of the EAACI/GA2 LEN/ $\mathrm{EDF} / \mathrm{WAO}$ guideline for the definition, classification, diagnosis, and management of urticaria. Allergy 2014;69:e1-e29.

5 Brockow K, Ring J: Emergency treatment and management of anaphylaxis. Hautarzt 2013; 64:81-87.

6 Simons FE, Ardusso LR, Bilo MB, et al: 2012 update: World Allergy Organization guidelines for the assessment and management of anaphylaxis. Curr Opin Allergy Clin Immunol 2012;12:389-399.
7 Society for Dermopharmacy: Guideline Dermatological Prescriptions (2013). http:// www.gd-online.de.

8 Scientific Institute of the Dutch Pharmacists: Prednisolondrank $1 \mathrm{mg} / \mathrm{ml}$, prednisolondrank $5 \mathrm{mg} / \mathrm{ml}$; in Formularium der Nederlandse Apothekers, ed 5 (in Dutch). The Hague, Royal Dutch Association for the Advancement of Pharmacy, 2013, pp 432-435.

9 Commission of the German Pharmaceutical Code/New German Formulary: Prednisolone oral solution $1 \mathrm{mg} / \mathrm{ml}$ or $5 \mathrm{mg} / \mathrm{ml}$ (NRF 34.1); in Federal Union of German Associations of Pharmacists: New German Formulary. Eschborn, Govi, 2012.

10 Ahmed M, Morrel EM, Clemente E: Bioavailability and pharmacokinetics of a new liquid prednisolone formulation in comparison with two commercially available liquid prednisolone products. Curr Ther Res 2001;62: 548-556.

11 US Food and Drug Administration: Bioequivalence - application No. 75-117. 1998. http:// www.accessdata.fda.gov/drugsatfda_docs/ anda/2000/75-117_Orapred_bioeqr.pdf.
12 Hi-Tech Pharmacal: Package insert (7/13): prednisolone sodium phosphate oral solution $5 \mathrm{mg} / 5 \mathrm{ml}$. http://www.akorn.com/documents/catalog/package_inserts/ 50383-040-04.pdf.

13 Pai Pharmaceutical Associates: Package insert (6/09): prednisolone sodium phosphate oral solution $15 \mathrm{mg} / 5 \mathrm{ml}$. http://www.paipharma. $\mathrm{com} / \mathrm{pdfs} / \mathrm{ffe} 6 \mathrm{efb} 0-\mathrm{a} 0 \mathrm{ae}-477 \mathrm{a}-\mathrm{b} 04 \mathrm{~b}$ c6def44091bb_insert.pdf.

14 Sanofi-Aventis Canada: Product monograph Pediapred - prednisolone sodium phosphate oral solution $15 \mathrm{mg} / 5 \mathrm{ml}$. http://www.products.sanofi.ca/en/pediapred.pdf.

15 Mission Pharmacal: Package insert (8/12) prednisolone sodium phosphate oral solution $25 \mathrm{mg} / 5 \mathrm{ml}$. http://www.akorn.com/documents/catalog/package_inserts / 50383-040-04.pdf.

16 Cancer Therapy Evaluation Program: Common Terminology Criteria for Adverse Events (CTCAE) - version 3.0. 2006. http:// www.ctep.cancer.gov.

17 Lucas-Bouwman ME, Roorda RJ, Jansman FG, Brand PL: Crushed prednisolone tablets or oral solution for acute asthma? Arch Dis Child 2001;84:347-348. 